\title{
EDUCAÇÃO EM SAÚDE COMO FERRAMENTA PARA A PREVENÇÃO DOS ACIDENTES DOMÉSTICOS COM CRIANÇAS: RELATO DE EXPERIÊNCIA
}

\section{Maria Ines dos Santos*1, Andreane Nathalia Pereira de Almeida Monteiro' ${ }^{1}$, José Romero Diniz1, Maria Amanda Lima Batista1, Raimundo Valmir de Oliveira², Cláudia Fabiane Gomes Gonçalves $^{2}$, Célia Maria Ribeiro de Vasconcelos ${ }^{2}$ and José Silvanderson Santos da Silva ${ }^{3}$}

${ }^{1}$ Enfermeiros. Instituto Federal de Educação, Ciência e Tecnologia (IFPE), Pesqueira/PE; ${ }^{2}$ Docentes. Curso Bacharelado em Enfermagem, IFPE, Pesqueira/PE; ${ }^{3}$ Docente. Escola Estadual Prefeito Alfredo de Araújo Grande, Arenápolis/MT

\section{ARTICLE INFO}

\section{Article History:}

Received $18^{\text {th }}$ October, 2020

Received in revised form

$14^{\text {th }}$ November, 2020

Accepted $06^{\text {th }}$ December, 2020

Published online $30^{\text {th }}$ January, 2021

\section{Key Words:}

Acidentes, Educação em saúde, Saúde da criança, Promoção da saúde, Saúde pública. *Corresponding author:

Maria Ines dos Santos,

\begin{abstract}
O projeto teve por objetivo promover educação em saúde para cuidadores de crianças do município de Pesqueira - PE. O estudo abordou a pedagogia da problematização como percurso metodológico, construindo o conhecimento a partir das situações-problemas vivenciadas pelos participantes. As oficinas foram desenvolvidas com gestantes e cuidadores de crianças adscritos a Estratégia Saúde da Família (ESF) das comunidades do CAIC, Pitanguinha, Xucurus e Angicos. Os diálogos com a população foram ferramentas poderosas para conhecermos a situação local, os entraves e conhecimento prévio de cada participante. A fala, exposição e construção conjunta das oficinas sobre os riscos do ambiente doméstico, as maneiras de prevenir os acidentes domésticos com crianças e a promoção do ambiente saudável para o crescimento seguro da criança, pôs os cuidadores como protagonista das suas experiências, fator importante para participação social nas ações de saúde e mudança de hábitos.
\end{abstract}

Copyright (C) 2021, Hugo Marques Galindo et al. This is an open access article distributed under the Creative Commons Attribution License, which permits unrestricted use, distribution, and reproduction in any medium, provided the original work is properly cited.

Citation: Maria Ines dos Santos, Andreane Nathalia Pereira de Almeida Monteiro, José Romero Diniz et al. 2021. "Educação em saúde como ferramenta para a prevenção dos acidentes domésticos com crianças: relato de experiência”, International Journal of Development Research, $11,(01), 43504-43507$.

\section{INTRODUCTION}

As crianças constituem o grupo mais vulnerável aos desastres e acidentes no estrato populacional (BRASIL, 2018; AGUIAR et al., 2020). Quanto mais jovem e imatura for a criança, menor sua percepção de risco e maior sua vulnerabilidade e dependência de terceiros, em termos de segurança contra acidentes e desastres (COSTA et al., 2017). No Brasil, um estudo realizado por Malta et al. (2015) sobre o perfil dos atendimentos de emergência ou acidentes e violência com crianças menores de 10 anos, constatou que 97,5\% das crianças foram vítimas de acidentes, sendo $67,4 \%$ no ambiente doméstico. Corriqueiramente, os acidentes são percebidos como situações inevitáveis, não desejadas pelas pessoas, porém, é um evento previsível e, portanto, passível de prevenção primária, através de medidas de prevenção que consideram o nível de desenvolvimento da criança e aspectos estruturais do ambiente (GURGEL; MONTEIRO, 2016; BARCELOS et al., 2018). O ambiente doméstico pode e deve ser um meio de promoção da saúde, entretanto, muitas vezes, torna-se propiciador de doenças e/ou agravos à saúde, como osacidentes, que revelam um grave problema de saúde pública (GHISI et al., 2018). Contudo, o acidente doméstico é uma das principais causas dos atendimentos, internações, incapacidades e óbitos em crianças e tem contribuído, de forma considerável, para manter elevada a taxa de morbimortalidade infantil (BRASIL, 2014; GHISI et al., 2018; FARIA et al., 2018). Os fatores de riscos encontrados no ambiente doméstico podem comprometer o desenvolvimento da criança, contribuindo para provocar diversos tipos de acidentes que, em determinados casos, podem acarretar graves lesões ou sequelas irreversíveis (RODRIGUES et al., 2015). Os principais tipos de acidentes domésticos encontrados são quedas, contusões, cortes, queimaduras, escoriações, esmagamentos, mordeduras e perfurações (BRASIL, 2014; GHISI et al., 2018). Tendo em vista que a criança passa a maior parte do seu tempo no domicílio, e que este ambiente é considerado um local de perigo para os acidentes, a supervisão em tempo integral por um adulto é de fundamental importância para a prevenção das ocorrências (GURGEL; MONTEIRO, 2016; RIBEIRO et al, 2019). Sendo assim, o cuidador da criança necessita de conhecimento referente à prevenção dos acidentes para usar as 
medidas adequadas e intensificar a atenção no cuidado com os perigos dentro de casa. Esse conhecimento pode ser adquirido através de capacitação e oficinas destinadas a aumentar a consciência e precauções tomadas a fim de evitar lesões em crianças (BRAGA et al., 2020). Nesta perspectiva, a educação em saúde é uma ferramenta com grande potencial para a promoção da saúde, prevenção e redução dos riscos de acidentes com crianças no ambiente doméstico, com consequente redução dos internamentos e gastos hospitalares, além de contribuir na redução dos índices de morbimortalidade na infância e deve ser realizada, com vistas à harmonia do crescimento e desenvolvimento saudável da criança. Dessa forma, o presente estudo objetivou contribuir para a redução desses agravos por meio da educação em saúde com gestantes e cuidadores de crianças do município de Pesqueira - PE, através de oficinas em saúde.

\section{METODOLOGIA}

A fim de alcançar os objetivos elencados, adotou-se como estratégia a metodologia da problematização, considerando que a proposta das oficinas foi a de construir o conhecimento a partir das situações problemas vivenciadas e relatadas pelos participantes. A utilização de metodologias centradas nos interesses e saberes dos participantes visa potencializar a autonomia e emancipação dos indivíduos, frente a problemas concretos vivenciados pelos sujeitos (BORDENAVE; PEREIRA, 1998). As oficinas foram norteadas pela metodologia da problematização desde a sua fase inicial, que incluiu reconhecimento do território das Estratégias de Saúde da Família (ESF), discussão de problemas encontrados, levantamento bibliográfico, elaboração de hipóteses de solução e implementação de ação (BORDENAVE; PEREIRA, 1998). A opção por esta estratégia metodológica buscou, também, integrar o graduando extensionista à proposta pedagógica adotada pelo Curso de Graduação em Enfermagem - Campus Pesqueira, que tem na problematização seu eixo metodológico, e no qual está vinculado esse projeto. Neste sentido, o referido projeto foi desenvolvido com dois públicos específicos: gestantes e cuidadores de crianças adscritos a ESF das comunidades do CAIC, Pitanguinha, Xucurus e Angicos do município de Pesqueira - PE, no período de março a novembro de 2016, resultado de um projeto de extensão. Estas comunidades foram escolhidas por sediarem atividades curriculares e de extensão do Curso de Graduação em enfermagem do IFPE - Campus Pesqueira e, por apresentarem vulnerabilidade sócio-econômico, como também, por já ter sido cenário de pesquisa sobre acidentes domésticos com crianças, caracterizando-se, assim, como um contexto de vivências de ensino, pesquisa e extensão.

As gestantes e os cuidadores de crianças destas localidades foram identificados a partir do cadastro existente nas unidades acima referidas, através dos dados secundários. O Agente Comunitário de Saúde (ACS) foi o elo que intermediou a comunicação para a realização das oficinas, visto que o planejamento das oficinas foi realizado em conjunto com as equipes da ESF. A partir desse ponto, se estabeleceu um plano de ação e a consecução das oficinas, com visitas ao contexto de vida das gestantes e cuidadores para levantamento de dados primários e reconhecimento do território, o qual subsidiou a fase de planejamento das oficinas. As referidas oficinas foram realizadas periodicamente, em um turno, com flexibilização do horário conforme a disponibilidade dos sujeitos participantes, obedecendo a um calendário e horários previamente construídos com as equipes da ESF, a fim de que, ao final do projeto, todas as unidades elencadas fossem contempladas com, no mínimo, uma oficina. Para o planejamento das oficinas, foram realizados encontros com todos os profissionais das equipes da ESF (enfermeiros, técnicos de enfermagem, Agentes Comunitários de Saúde, médicos e dentistas), a fim de realizar uma escuta sobre a problemática em tela e, posteriormente, planejar as oficinas para o público alvo. Assim, foi possível identificar o perfil da comunidade a partir da experiência dos profissionais. Posteriormente, foram elaboradas questões norteadoras que permitiram, por meio das respostas, colher as percepções que os profissionais tinham sobre acidentes domésticos com crianças, adequando assim o planejamento das oficinas subsequentes ao contexto local.

As questões norteadoras aplicadas aos profissionais foram as seguintes: 1) Qual a sua percepção de um acidente com crianças? 2) Quais os principais tipos de acidentes que acometem crianças na comunidade? 3) Quais os principais riscos que você identifica no ambiente doméstico que pode levar a criança a um acidente? 4) Qual a conduta adotada no atendimento a criança vítima de acidente? 5) De que forma é trabalhada a prevenção de acidentes com crianças?

As questões foram exploradas uma de cada vez, em pequenos grupos, que eram estimulados à reflexão crítica da temática, suscitada pela pergunta norteadora. Em seguida, cada integrante registrou suas impressões em um grande painel. Portanto, a partir das oficinas com os profissionais $\mathrm{e}$ considerando as informações recolhidas do material produzido nos respectivos encontros foi realizado o planejamento das oficinas subsequentes, direcionadas as gestantes e cuidadores. Nestas oficinas, era proposto aos participantes uma temática, a partir da qual, eram estimulados a se expressarem sobre o tema. Em pequenos grupos, eram orientados a construírem um painel que expôs seus conhecimentos sobre o tema. Assim, ao final, a construção era socializada com o grande grupo, enriquecido com a vivência dos participantes. As temáticas sugeridas foram: Origem e causas dos acidentes infantis; Prevenção de acidentes domésticos; Conhecendo o ambiente doméstico; Análise de dados locais de incidência de acidentes com crinaças. Por fim foi construído coletivamente, ao final de cada oficina, um plano de enfrentamento da problemática local, com a confecção de um folder informativo sobre prevenção de acidentes com crianças.

As oficinas foram sempre precedidas de uma dinâmica, objetivando estimular a integração, o conhecimento mútuo e a participação. As dinâmicas foram inseridas para facilitar o processo de ensino aprendizagem, antes e durante as atividades propostas para o grupo, valorizando sempre o diálogo, a escuta e o reconhecimento de saberes pré-existentes. As ações desenvolvidas durante as oficinas, consideraram as especificidades dos planos de trabalhos dos bolsistas: acidentes domésticos com crianças para gestantes; educação em saúde para cuidadores de crianças; acidentes domésticos por trauma em crianças; acidentes domésticos por ingesta de produtos químico e bronco aspiração com crianças e primeiros socorros.

\section{RESULTADOS E DISCUSSÃo}

Foram realizadas 09 oficinas, sendo que, quatro foram direcionadas aos profissionais das ESFs e cinco para gestantes e cuidadores de crianças de 1 a 5 anos de idade. 
Oficinas para os profissionais das Estratégias de Saúde da Família (ESF): Quanto aos profissionais de saúde, 32 participaram das oficinas, entre enfermeiros e técnicos de enfermagem. Apesar do convite, não houve participação de médicos ou dentistas. Na tabela 1 é possível visualizar a distribuição dos profissionais que participaram das oficinas, de acordo com unidade de saúde e nível de escolaridade.

Tabela 1. Distribuição dos participantes da equipe de saúde quanto à unidade de saúde e nível de escolaridade, $\mathbf{N}=32$

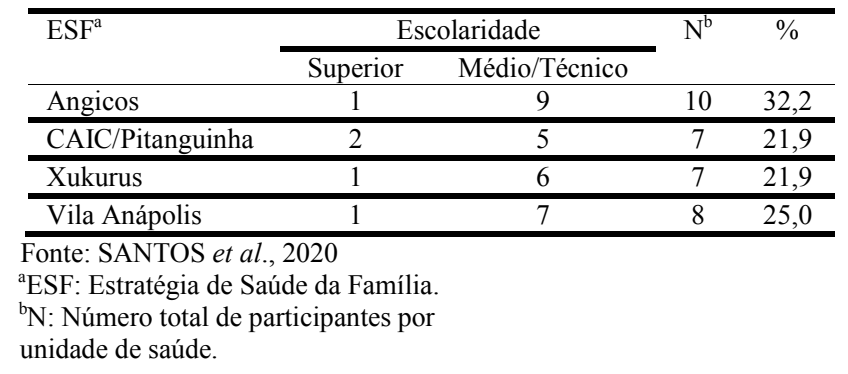

É possível perceber na tabela 1, um maior número de participantes de nível médio e técnico. Esse quantitativo refere-se aos ACS, que tiveram presença expressiva em todas as oficinas, que reflete $o$ grande quantitativo desses profissionais nas unidades básicas de saúde. A Política Nacional de Atenção Básica preconiza que o número desse profissional deve ser suficiente para cobrir $100 \%$ da população cadastrada, com no máximo 750 pessoas para cada ACS, e de 12 ACS por equipe de Saúde da Família (BRASIL, 2017). O ACS adentra nos lares e conhecem com maior propriedade a realidade local (SPERONI et al., 2016), por este motivo é o primeiro a identificar eventos que ensejam as crianças em acidentes domésticos. Nas discussões coletivas, constatou-se a inexistência de ações preventivas contra os acidentes com crianças no ambiente doméstico. Relato que marcadamente evidenciou a importância do projeto por possibilitar uma sensibilização para a temática em tela por parte dos profissionais. Nesta perspectiva, a ESF inserida na atenção primária, possui subsídios para intervir na problemática acima exposta, desde que suas ações estejam em conformidade com as diretrizes do Sistema Único de Saúde (SUS), que preceitua entre outras, a integralidade das ações, desenvolvendo atividades de promoção, prevenção, tratamento e reabilitação da saúde da população adscrita. Segundo os pressupostos desta estratégia, a atenção deve estar centrada na família, entendida e percebida a partir de seu ambiente físico e social, o que possibilita às equipes de Saúde da Família uma compreensão ampliada do processo saúde-doença e da necessidade de intervenções que vão além da prática curativa (BELTRAME et al., 2020).

Oficinas para gestantes e cuidadores de crianças: Nas oficinas destinadas à comunidade adstrita nas unidades de saúde, participaram 120 pessoas, dentre gestantes e cuidadores de crianças de 1 a 5 anos de idade. Foram realizadas cinco oficinas no total, conforme disposto na Tabela 2. No decorrer das oficinas, alguns participantes relataram já ter passado pela experiência dos seguintes acidentes com crianças no seu convívio familiar: quedas, ingestão de produtos tóxicos, queimaduras, engasgo com pequenos objetos, ingestão de medicamentos e choque elétricos. Destes, os acidentes que foram comuns às quatros comunidade participantes das oficinas foram quedas, queimaduras e engasgos, o que corrobora com outros estudos (RODRIGUES et al., 2015;
COSTA et al., 2017; FARIA et al., 2018; BRAGA et al., 2020).

Tabela 2. Distribuição das gestantes e cuidadores de crianças que participaram das oficinas, $\mathbf{N}=120$

\begin{tabular}{llll}
\hline OFICINA & ESF $^{\mathrm{a}}$ & $\mathrm{N}^{\mathrm{b}}$ & $\%$ \\
\hline Oficina 1 & CAIC/Pitanguinha & 30 & 25,0 \\
\hline Oficina 2 & Xucurus & 27 & 22,5 \\
\hline Oficina 3 & Angicos & 25 & 20,8 \\
\hline Oficina 4 & CAIC & 18 & 15,0 \\
\hline Oficina 5 & Pitanguinha & 20 & 16,7 \\
\hline Fonte: SANTOS et al., 2020 & & \\
a ESF: Estratégia de Saúde da Família. \\
b N: Número total de participantes por unidade de saúde.
\end{tabular}

De acordo com um estudo realizado em um hospital de referência pediátrica em São Luiz, no Maranhão, as quedas representaram cerca de $60 \%$ dos acidentes envolvendo crianças (BRAGA et al., 2020). Para Brito e Rocha (2015), estes acidentes estão relacionados ao comportamento da família, sua rede social, aos fatores educacionais, econômicos, sociais e culturais, como também as fases de vida específicas da criança, caracterizadas pela curiosidade aguçada e aprendizado contínuo. A equipe de saúde tem um papel fundamental na promoção de ações em saúde, por estar em contato contínuo com os pais de crianças atendidas nas unidades de saúde, pois através de orientações bem direcionadas é possível reduzir o número de agravos provocados pelos acidentes domésticos nas crianças (BARCELOS et al., 2018; ROMA et al., 2018). Por outro lado, a Política Nacional de Redução da Morbimortalidade por Acidentes e Violências, publicada no ano de 2001, estabelece que "a promoção da saúde deve embasar todos os planos, programas, projetos e atividades da redução da violência e acidentes" e reforça que a prevenção primária deve ser estimulada, fortalecendo os indivíduos para melhorar suas condições de vida e de saúde, ao desenvolver suas aptidões pessoais (BRASIL, 2001). Nesta perspectiva, a educação em saúde é uma ferramenta com grande potencial para a promoção da saúde, prevenção e redução dos riscos de acidentes com crianças no ambiente doméstico, com consequente redução dos internamentos e gastos hospitalares; como também reduz os índices de morbimortalidade na infância, e deve ser realizada, com vistas à harmonia do crescimento e desenvolvimento saudável da criança. Foi nesta perspectiva que o presente projeto foi desenvolvido em formato de oficinas, a fim de promover o mais precocemente possível à educação em saúde para as gestantes e cuidadores de crianças do município de Pesqueira - PE, a fim de reduzir os danos causados às crianças, e proporcionar um crescimento pleno, de todas as potencialidades, em vista da harmonia do ser humano com o seu micro e macroambiente, estimulando relações de bem-estar e crescimento saudável.

\section{Conclusão}

A adoção da educação em saúde durante as oficinas superaram a simples função de difundir conceitos e comportamentos, foi muito mais além, possibilitando problematizar, em uma discussão horizontal, as situações concretas vivenciadas pelos sujeitos. As atividades lúdicas atuaram como um recurso privilegiado para explorar os significados culturais reconhecidos e valorizados no contexto de cada participante das palestras e, por isso, constitui-se como importante dispositivo para o desenvolvimento da consciência crítica e da autonomia dos envolvidos. Os diálogos com a população 
foram ferramentas poderosas para conhecermos a situação local, os entraves e conhecimento prévio de cada participante. A troca de saberes entre estudantes e participantes das oficinas nos aproxima da realidade local, criando um vínculo construtivo de confiança, fundamental nas relações entre os profissionais e a comunidade. A fala, exposição e construção conjunta das oficinas sobre os riscos do ambiente doméstico, bem como as maneiras de prevenir os acidentes domésticos com crianças e a promoção do ambiente saudável para o crescimento seguro da criança, pôs os cuidadores como protagonista das suas experiências, fator importante para participação social nas ações de saúde e mudança de hábitos.

\section{REFERÊNCIAS}

Aguiar, K.V. da C.S., Cruz, R. da C., Silva, R.T. de A., Bofim, A.S. (2020). Intoxicação exógena acidental em crianças no estado da Bahia: 2013 a 2017. Revista Eletrônica Acervo Saúde, v. 12, n. 11, pp. e3422.

Barcelos, R.S., Del-Ponte, B., Santos I.S. (2018) Interventions to Reduce Accidents in Childhood: A Systematic Review. Jornal de Pediatria, v. 94, n. 4, pp. 351-67.

Beltrame, I.L., Cincotto, F., Makabe, M.L. (2020) Percepções dos agentes comunitários de saúde (ACS) sobre a importância do estágio em saúde da família na formação médica. International Journal of Health Management Review, v. 6, n. 2, pp. 1-10.

Braga, L.C., Silva, A.C.O., Santos, G.F.L dos, Soares G.R. de O., Colins J.R.P., Santos, D.M.A. dos. (2020) Acidentes infantis atendidos nos hospitais públicos de referência pediátrica. R pesq cuid fundam online, v. 12, pp. 12081214.

Brasil. Ministério da saúde. (2017) Portaria n² 2.436, de 21 de setembro de 2017. Aprova a Política Nacional de Atenção Básica, estabelecendo a revisão de diretrizes para a organização da Atenção Básica, no âmbito do Sistema Único de Saúde (SUS). Atribuições dos profissionais da atenção básica. Disponível em: https://bvsms.saude.gov.br /bvs/saudelegis/gm/2017/prt2436_22_09_2017.html

Brasil. Ministério da Saúde. (2005) Política Nacional de Redução da Morbimortalidade por Acidentes e Violências. 2a edição. Portaria GM/MS n. 737, de 16 de maio de 2001. Disponível em: http://bvsms.saude.gov. br/bvs/publicacoes/politica_reducao_morbimortalidade_a cidentes_2ed.pdf

Brasil. Ministério da Saúde. (2018) Política Nacional de Atenção Integral à Saúde da Criança: orientações para implementação. Secretaria de Atenção à Saúde. Departamento de Ações Programáticas Estratégicas. Brasília, 180p. Disponível em: https://portal deboaspraticas.iff.fiocruz.br/wp-content/uploads/2018/ 07/Pol\%C3\%ADtica-Nacional-de-Aten $\%$ C3\%A7\% C3\%A3o-Integral-\%C3\%A0-Sa\%C3\%BAde-daCrian\%C3\%A7a-PNAISC-Vers\%C3\%A3oEletr\%C3\%B4nica.pdf
Brasil. Ministério da Saúde (2014) Acidentes domésticos ainda são principal causa de morte de crianças até 9 anos. Portal do Governo Brasileiro. Brasília. Disponível em: https://www.gov.br/saude/pt-br

Brito, M.A., Rocha, S.S. (2015) A criança vítima de acidentes domésticos sob o olhar das teorias de enfermagem. Rev. de Pesquisa Cuidado é Fundamental Online. pp. 33513365.

Costa, S.N.G. da; Silva, J.M.M. da, Freitas, B.H.B.M. de, Reis, A.F.C. (2017) Acidentes infantis: conhecimento e percepção de educadoras de creches. Rev enferm UFPE online, v. 11, n. 10, p. 3845-52.

Faria, C.G. de, Queiroz, D.B., Matias, O., Melo, T.P. de. (2018) Principais causas de internação por acidentes domésticos na infância em um hospital universitário do oeste do Paraná. Brazilian Journal of Surgery and Clinical Research, v. 22, n. 2, p. 103-109.

Ghisi, G.C., Dias Júnior, G., Fachini, J.S., Santos Júnior, J.R. dos, Santos, T.C dos. (2018) Perfil epidemiológico das internações por acidentes domiciliares em um hospital pediátrico da região sul do Brasil. Arq Catarin Med, v. 47, n. 4, p. 29-38.

Gurgel, A.K.C., Monteiro, A.I. (2016) Prevenção de acidentes domésticos infantis: susceptibilidade percebida pelas cuidadoras. J res fundam care oline, v. 8, n. 4, p. 51265135.

Malta, D.C., Mascarenhas, M.D.M., Neves, A.C.M. das, Silva, M.A. da. (2015) Atendimentos por acidentes e violências na infância em serviços de emergências públicas. Cad Saúde Pública, v. 31, n. 5, p. 1095-1105.

Reichert, A.P. da S., Leônico, A.B. de A., Toso, B.R.G., Santos, N.C.C. de B., Vaz, E.M.C., Collet, N. (2016) Orientação familiar e comunitária na Atenção Primária à Saúde da criança. Ciênc saúde colet, v. 21, n. 1, p. 1-10.

Ribeiro, M.G.C., Paula, A.B.R., Bezerra, M.A.R., Rocha, S.S., Avelino, F.V.S.D., Gouveia, M.T.O. (2019) Determinantes sociais da saúde associados a acidentes domésticos na infância: uma revisão integrativa. Rev Bras Enferm, v. 72, n. 1.

Rodrigues, L.M. de C., Moura, M.E.B., Melo, T.M.T. de C., Silva, M.N.P. da, Alencar, G.C. de A.A., Silva, L.M.M. (2015) Atualização sobre a ocorrência de acidentes envolvento crianças. Rev enferm UFPE online, v. 9, n. 9, p. 1028-34.

Roma, K.M. dos S., Gomes, M.F.P., Reticena, K. de O., Capellini, V.K., Fracolli, L.A. (2018) Prevenção de acidentes na primeira infância na estratégia saúde da família: perspectiva dos pais. Rev Enferm UFPI, v. 7, n. 2, p. 28-34.

Speroni, K.S., Fruet, I.M.A., Dalmolin, G. de L., Lima, S.B.S. de. (2016) Percepções dos agentes comunitários de saúde: contribuições para a gestão em saúde. Rev Cuid, v. 7, n. 2, p. 1325-37. 